\title{
Factores asociados al rendimiento académico en adultos
}

\section{Associated factors to academic performance in adult}

\section{Fatores associados no rendimento escolar em adultos}

\author{
Mawency Vergel-Ortega ${ }^{1}$ \\ José Joaquín Martínez-Lozano² \\ Sandra Liliana Zafra-Tristancho ${ }^{3}$
}

Fecha de recepción: mayo 2016

Fecha de aceptación: junio 2016

Para citar este artículo: Vergel-Ortega, M.; Martínez-Lozano, J.J. y Zafra-Tristancho, S.L. (2016). Factores asociados al rendimiento académico en adultos. Revista Científica, 25, 206-215. Doi: 10.14483/udistrital.jour.RC.2016.25.a4

\begin{abstract}
Resumen
El artículo tiene como objetivo identificar los factores asociados al rendimiento académico en estudiantes adultos, en módulos de matemáticas y estadística. Se realizó un estudio cuantitativo correlacional en una muestra de 80 estudiantes con edades superiores a 30 años. Un grupo de factores de aspectos institucionales, sociodemográficos, psicosociales y pedagógicos se emplearon como variables independientes. Resultados indican que el estilo de aprendizaje asociado a tipo de inteligencia, conciencia de déficit asociada a capacidad de abstracción y motivación de la familia predicen niveles más altos de rendimiento constituyéndose en variables explicativas. Como conclusión se esgrime que el tipo y estilo de aprendizaje, tipo de inteligencia, motivación, conciencia de déficit son factores asociados al rendimiento en adultos.
\end{abstract}

Keywords: rendimiento académico, adultos, universidad, matemáticas, motivación familiar.

\begin{abstract}
The article has the objective identify the associated factors to academic performance of adult students in mathematics and statistics modules. Was realized a study quantitative correlational in a sample of 80 students with age over 30 years old. A group of institutional aspects, social-demographics, psychosocials and pedagogic factors were used as independent variable. Results indicate that style of learning associated with type of intelligence, deficit conscience associated to abstraction capability and family motivation predicts the higher levels of performance, constituting explicative variables. Conclusion: type and style of learning, type of intelligence, motivation, conscience of deficit are associated factors to performance in adults.
\end{abstract}

Palabras Clave: academic performance, adults, university, mathematics, family motivation.

Universidad Francisco de Paula Santander. Cúcuta. Colombia. Contacto: mawency@ufps.edu.co

Universidad Francisco de Paula Santander. Cúcuta. Colombia. Contacto: josemartinez@ufps.edu.co

Policía Nacional de Colombia. Bogotá, Colombia. Contacto: sandra.zafra@correo.policia.gov. 


\section{Resumo}

$\mathrm{O}$ artigo tem como objetivo identificar os fatores associados com os alunos adulto acadêmicos em módulos de Matemática e Estatística . um estudo quantitativo de correlação foi realizado sobre uma amostra de 80 estudantes acima de 30 anos de idade. Um grupo de fatores institucionais, sócio- demográficos, psicossociais e educacionais foram utilizadas como variáveis independentes. Os resultados indicam que o estilo de aprendizagem associadas ao tipo de inteligência , consciência dos déficits associados com capacidade de abstração e motivação da família prever os níveis mais altos de desempenho tornando-se variáveis explicativas. Conclusão: tipo e estilo de aprendizagem, tipo de inteligência, motivação, déficits da consciência são fatores associados ao desempenho em adultos.

Palavras chave: desempenho acadêmico , adultos , universitários, matemática, motivação família .

\section{Introducción}

La disincronía entre edad cronológica y edad mental en estudiantes de programas de educación superior jornada nocturna, respecto a estudiantes de jornada diurna, implica que tengan diferente desarrollo en el ámbito emocional, social y cognitivo; teniendo en cuenta, además, que su rendimiento académico es una problemática que enfrentan los docentes en cursos con alumnos de diferentes edades, género y ocupación, en especial en educación universitaria (Erazo, 2013; Martin, Wilson, Arief y Ginns, 2013).

De otra parte, cuando un docente asume un curso, prepara un plan de clase para orientar a sus estudiantes con la misma metodología y las mismas estrategias pedagógicas. No obstante, al llegar al aula e iniciar los procesos pedagógicos encuentra estudiantes con alto y bajo rendimiento, algunos interpretan rápidamente resultados, otros no, y algunos estudiantes no llegan a adquirir las competencias básicas exigidas en el programa.

Múltiples estudios buscaron identificar predictores del rendimiento, explorando variables sociodemográficas y factores psicológicos del estudiante
(Garbanzo, 2013); mientras algunos se centraron en variables asociadas a recursos institucionales (Bartual y Poblet, 2009), otros relacionaron rendimiento con antecedentes de estudiantes exitosos en grados previos (Fernández, 2012) y con locus control como localización de causas de eventos en la vida de las personas (Mayora y Fernández, 2015), pero son escasas las investigaciones en adultos que cursan programas universitarios.

En cuanto a los adultos que cursan programas de formación profesional en administración pública, en jornada nocturna, estos cuentan con características especiales, a saber: edades superiores a 30 años, $95 \%$ trabaja, $20 \%$ lidera empresas. Aquí es importante conocer su capacidad de razonamiento abstracto: si cuentan con estilos de aprendizaje y habilidades en ciertas áreas afines a la matemática, y si rasgos cognitivos, afectivos y fisiológicos se constituyen en indicador de cómo perciben, interaccionan y responden a ambientes de aprendizaje. Entendida como capacidad de razonamiento, la facultad humana que permite la resolución de problemas y la capacidad de abstracción, implicaría extrapolar una situación vivida previamente a un problema concreto a resolver (Ortega et al., 2014). Así mismo, una correcta evaluación de las capacidades cognitivas del adulto requerirá una serie de consideraciones previas, como: adaptar la exploración al sujeto que se va a evaluar; seleccionar pruebas adecuadas, capacidades físicas y psicológicas del sujeto, nivel de inteligencia premórbido, experiencia, características médicas y sociales del adulto, donde sistema perceptivo, atención subjetiva o condicionada a gustos o intereses (Alegret et al., 2012; Horton y Hartlage, 2010).

La ausencia de líneas de trabajo suficientemente contrastadas en rendimiento académico en adultos, sus cambios en la realidad sociodemográfica y cultural en los últimos años y la aparición de nuevos escenarios de enseñanza y aprendizaje planteados como espacios estructurados de formación, con un currículo formalizado y estable, hacen especialmente oportuno, y pedagógicamente 
necesario, disponer de mayor información sobre el modo en que aprenden, de manera que se realicen mejores diseños didácticos y metodologías acordes a las características específicas de las personas mayores de 30 años.

Con referencia a lo planteado por Krusche (2006) adaptarse a cada estudiante y a su forma de aprender, e identificar tanto el perfil educacional del estudiante mayor (valorar las fortalezas y debilidades) como sus talentos y patrones de aprendizaje, complementa los planes educacionales que buscan su total desenvolvimiento.

En este ámbito, Gardner (1983) por su parte, sostuvo que existe un conjunto de competencias humanas relativamente autónomas que conforman la estructura intelectual del hombre, inteligencias que pueden ser modeladas y combinadas de múltiples maneras en edades tempranas. Sin embargo, en los adultos, la capacidad intelectual, liderazgo y otros valores de personas que han triunfado en los negocios, o en los deportes, requiere utilizar un tipo de inteligencia o destreza a desarrollar en cada individuo de diferente manera, partiendo de sus capacidades y aprovechando sus puntos fuertes. Potencialidades desarrolladas dependiendo del medio ambiente, las experiencias vividas, la educación recibida, apoyados en sus capacidades y motivación.

Gardner identificó ocho tipos distintos de inteligencia en todos los seres humanos en mayor o menor medida, a saber: lingüística, lógico-matemática, espacial, musical, corporal-cinestésica, intrapersonal, interpersonal, naturalista. Además, tendría que plantearse si una educación centrada en tipos de inteligencia es la más adecuada para preparar estudiantes adultos para vivir en un mundo cada vez más complejo cuyos avances tecnológicos surgen a gran velocidad.

A partir de las características del adulto, en este artículo se determinaron factores asociados, la relación existente entre el tipo de inteligencia de los estudiantes mayores de 30 años, su estilo de aprendizaje y los resultados de rendimiento académico obtenido en cursos de matemáticas y estadística en estudiantes de Administración Pública. Se determinaron variables que inciden en el aprendizaje según el modelo por situaciones problema, dando respuesta a la pregunta “¿Qué factores están asociados al rendimiento académico de los estudiantes?". Para ello se busca responder: "En una escala de inteligencia, ¿cuál es la capacidad de razonamiento abstracto de los estudiantes adultos?", ¿Qué tipos de aprendizaje, estilo e inteligencias múltiples poseen los estudiantes mayores de 30 años?", “CCómo es el rendimiento académico de estudiantes de Administración Pública?", "¿Qué variables se relacionan o explican el rendimiento académico en matemáticas y estadística en los estudiantes mayores de 30 años de Administración Pública?".

\section{Metodología}

El estudio se enmarca en el enfoque cuantitativo, de tipo campo, diseño correlacional, a 80 estudiantes mayores de 30 años que cursan asignaturas de matemáticas y estadística en programas de pregrado de administración pública. Las variables independientes consideradas fueron: edad, género, percepción de agrado o dificultad hacia la asignatura, índice socioeconómico, expectativa de logro, motivación familiar, planta física e índice de servicios institucionales, estrategia metodológica, dominio del tema del docente, índice de percepción de relaciones socioafectivas, exigencia académica por parte del docente, tipo de inteligencia y estilo de aprendizaje.

También se consideraron variables relacionadas con el nivel de aprendizaje, mediante instrumento dirigido al docente, entre ellas: género, dominio de temas, grado académico, actualización, importancia brindada a la capacitación, metodología, exigencia académica, recursos para el desarrollo de la clase, frecuencia con que se asigna trabajos y tiempo destinado a la asignatura, así como actividades que se realizan en torno a ellas.

El razonamiento abstracto se valoró a través del subtest de semejanzas de la escala de inteligencia 
de Wechsler para adultos (WAIS-III), la cual midió la capacidad de converger en una sola idea dos elementos aparentemente distintos que guardan alguna relación común (Wechsler, 1999). Se evaluó la conciencia de déficit mediante las preguntas: "¿Cómo está su memoria?" y “¿Qué le preocupa?".

Se aplicó el cuestionario de inteligencias múltiples de Howard Gardner y el inventario de estilos de aprendizaje de Kolb, los cuales permitieron identificar características personales del estudiante respecto a la forma como procesaron la información (Muldoon y Weisberg, 2011). Para responder interrogantes como: "¿Cuáles son las maneras de aprendizaje de estos estudiantes?" y "¿Cómo perciben con mayor eficiencia la información?", se aplicó un instrumento (test) de 15 preguntas, con base en las respuestas dadas por los estudiantes.

Para verificar supuestos de análisis factorial, se utilizó la prueba complementaria de medida de adecuación muestral de Kaiser-Meyer-Olkin (KMO) que representa la correlación parcial de dos variables eliminando el efecto de las restantes; esta medida osciló entre 0 y 1 , valores menores de 0,50 se consideraron inaceptables, y para aquellos próximos o superiores a 0,70 se aconsejó la aplicación del análisis factorial, los valores mayores a 0,80 fueron deseables.

El análisis de componentes principales se utilizó como técnica basada en las correlaciones de las variables analizadas; lo deseable es que exista una correlación adecuada y no muy alta, pues puede causar el fenómeno denominado multicolinealidad. Para la consideración del número óptimo de factores, se tomó como indicador importante la magnitud de los autovalores (superior a 1); en este caso, se asumió el porcentaje de varianza explicada para cada factor y la cantidad de factores teóricos presentes, con lo cual se estableció un número fijo de factores a extraer fijado en 5. Las cargas factoriales representaron el peso de cada variable, para los factores obtenidos en la solución factorial; se consideró que la variable observada satura mejor en el factor, cuya carga factorial es más alta. Se realizó el análisis factorial confirmatorio para establecer el modelo estructural que definía los factores asociados (Martínez, Vergel y Zafra, 2016).

\section{Resultados}

Puntuaciones estandarizadas del subtest de semejanzas, del WAIS-III abreviado por rangos de edad, mostraron cómo puntuaciones para adultos mayores de 30 años osciló entre 11,5 y 13 puntos en promedio con una desviación estándar de 1,6; sensibilidad $\mathrm{SE}=79$, especificidad $\mathrm{SP}=67$, por lo cual los estudiantes se encontraron en un rango normal de inteligencia en prueba de razonamiento abstracto, teniendo en cuenta edad y estudios previos (Alegret et al., 2013).

El análisis de efecto del diagnóstico y conciencia de déficit, sobre el rendimiento en razonamiento abstracto mediante modelo lineal, mostró preservación de conciencia de déficit (90 \%, p < 0,0001), así mismo, estudiantes con conciencia de déficit tuvieron un mejor rendimiento; la conciencia de déficit se asoció a una mayor capacidad de abstracción, ya que se encontró un efecto estadísticamente significativo tanto de conciencia de déficit $(\mathrm{F}=58.703 ; \mathrm{p}<0,001)$ como del diagnóstico ( $\mathrm{F}$ $=3.417 ; p<0,001)$, pero no de interacción.

Los estudiantes ubicados en este nivel, tuvieron alta probabilidad de lograr competencias correspondientes al nivel básico de la asignatura matemática y de la asignatura estadística, resolviendo acertadamente ítems relacionados con la identificación de elementos básicos en contenidos de matemáticas y estadística, operaciones con conjuntos, funciones, determinación de medias, desviación, porcentajes. Referente al dominio de la comprensión lectora, su nivel fue bajo; en relación con el dominio de la expresión gráfica, los estudiantes tuvieron altas posibilidades de graficar (95 $\%, p<0,0002)$ pero baja capacidad para interpretar resultados y gráficas al enfrentarse a una situación aplicada.

De otra parte, el índice socioeconómico del estudiante, percepción de agrado o dificultad hacia 
la asignatura, estado de la planta física ( $\mathrm{t}=0,96$, $p=0,339)$, índice de percepción de relaciones socioafectivas $(t=-0,73, p=0,47)$, edad ( $\mathrm{e}>30$ años, $t=1,18, p=0,28)$ y género no dio evidencia de asociación ( $z=-0,9729, p=0,332)$. De acuerdo con los datos, hubo relación entre la expectativa de logro que tiene la familia del estudiante y su rendimiento académico. De manera que, al controlar las variables, cuando la expectativa de logro de la familia aumentó (1 punto), los estudiantes obtuvieron en promedio 0,17 puntos más en el rendimiento de la asignatura $(z=-2,83, p=$ $0,000)$. Así mismo, existió relación entre el rendimiento académico del estudiante y la motivación recibida por parte de su familia (aumento de nota en promedio 0,18 puntos más en la asignatura, $z=$ $-2,89, p=0,0001)$.

En concordancia con los datos obtenidos, al aumentar en una unidad el índice de recursos disponibles en el aula, en promedio y manteniendo constantes las otras variables, los estudiantes obtuvieron 0,5 puntos más en su rendimiento académico $(z=2,04, p=0,021)$. De igual manera, al aumentar en una unidad la escala de percepción del estudiante sobre el dominio que tiene el docente de la asignatura, estos aumentaron en 0,03 puntos más su rendimiento académico $(z=-2,53, p=0,01)$. Y, al aumentar en una unidad la percepción del estudiante sobre la exigencia académica del docente, los estudiantes incrementaron su rendimiento académico. Los alumnos percibieron la asociación entre experiencia del docente con títulos obtenidos y su dominio del tema.

Analizando el tipo de aprendizaje (figura 1), los estudiantes tuvieron un marcado dominio por el aprendizaje auditivo (60\%, p < 0,05); en $25 \%$ de ellos predomina el aprendizaje visual y el kinestésico; en 51,25\% ( $p<0,01)$ domina el aprendizaje kinestésico. Se destaca que algunos tuvieron un aprendizaje auditivo, pero el visual y el kinestésico no fueron representativos en ellos, mientras que hubo quienes estuvieron presentes los tres tipos de aprendizaje.

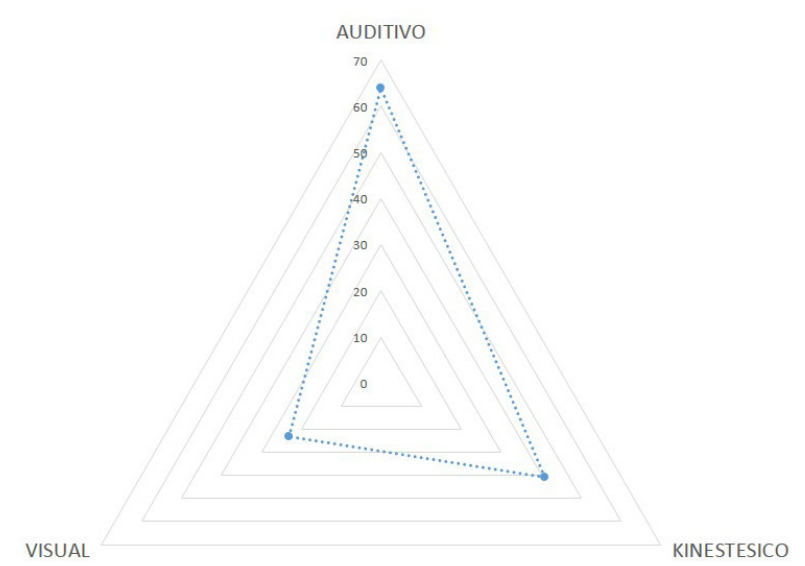

Figura 1. Tipos de aprendizaje presentes en estudiantes universitarios con edad mayor a 30 años.

Fuente: elaboración propia.

En cuanto al estilo de aprendizaje, en el análisis de la información (figura 2), se observó tendencia a puntajes altos en el estilo pragmático; no obstante, en su gran mayoría los estudiantes adultos, han hecho uso del estilo reflexivo (16 puntos, 41,25\%, $\mathrm{p}<0,01)$; la dispersión de los datos respecto a su valor promedio fue de 2,80, lo cual indicó que los estudiantes tuvieron buen proceso formativo.

En contraste, se observó que el estilo activo (12 puntos, $18,7 \%, \mathrm{p}<0,01$ ) fue el menos utilizado (figura 2), es decir, los estudiantes mostraron poco interés por la experiencia directa, y por la realización de nuevas tareas. Se observó la preferencia, en menor escala, por el estilo teórico (14,4 puntos, 18,7 $\%, p<0,05)$, y el pragmático (16 puntos, 31,25 \%, $\mathrm{p}<0,05)$; el primero consecuencia de un proceso de conceptualización abstracta y de deducción de conclusiones, proceso que requirió que fuesen metódicos, lógicos, objetivos, críticos, estructurados; y el segundo, les significó arriesgarse a implementar aplicaciones, lo cual, según manifiestos, les generó temor por aproximarse a nuevas teorías o a fracasar en el logro de este objetivo.

De otra parte, datos asociados con el tipo de inteligencia dominante en cada estudiante (tabla 1) permitieron determinar que las inteligencias intrapersonal e interpersonal fueron las más 
significativas con $78,3 \%$ y $69,6 \%$, respectivamente $(p<0,01)$. Le siguieron, en su orden, la inteligencia verbal/lingüística, musical/rítmica, lógica/ matemática, visual/espacial y, por último, la kinestésica/corporal. Un 26,1 \% de los estudiantes se identificaron con dos tipos de inteligencia; 39, $1 \%$, con tres tipos de inteligencia; 8,7 \% poseen cuatro tipos de inteligencia; así mismo, $13 \%$, cinco tipos de inteligencia; $4,3 \%$ reconocieron un tipo de inteligencia, y 4,3\% manifestaron presencia de seis tipos de inteligencia.

En general, los estudiantes poseen tres o más tipos de inteligencia y alcanzaron un nivel de desempeño alto en el aprendizaje: $24 \%$, con inteligencia verbal; $55 \%$, con inteligencia lógico-matemática; $28 \%$, con inteligencia visual espacial; $35 \%$, kinestésico corporal; $23 \%$, con inteligencia musical; $30 \%$, interpersonal, y $20 \%$, intrapersonal.

Estudiantes con inteligencia interpersonal e intrapersonal tuvieron rendimiento medio, aquellos con inteligencia lógico-matemático alcanzaron un rendimiento superior, y otros con inteligencia kinestésico-corporal, musical, verbal, visual, presentaron rendimiento bajo en matemáticas y estadística.

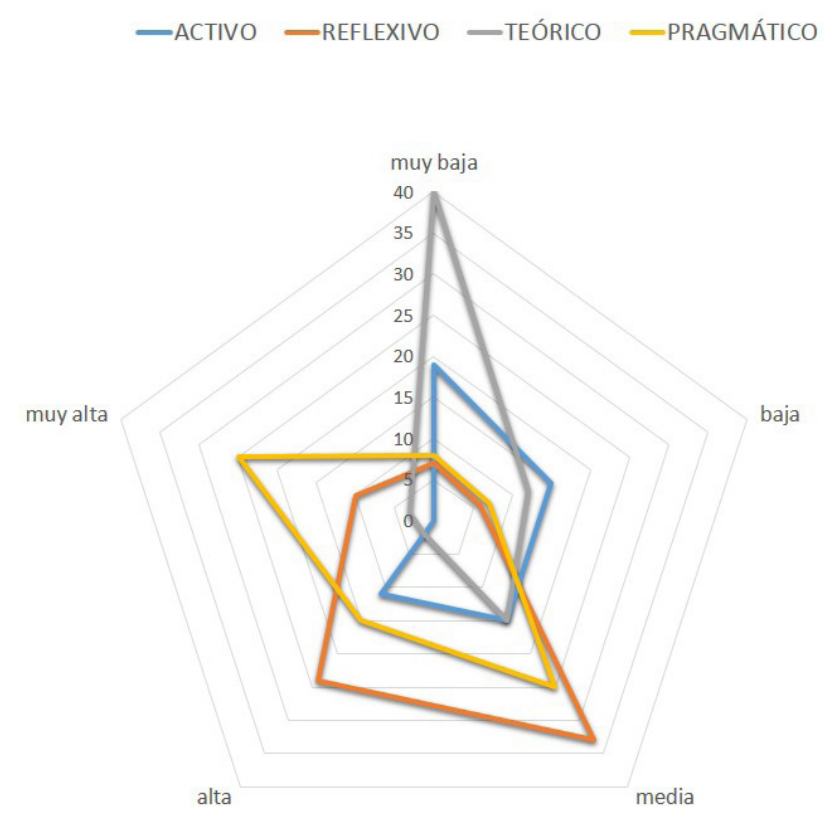

Figura 2. Estilos de aprendizaje presentes en estudiantes universitarios con edad mayor a 30 años.

Fuente: elaboración propia.

De otra parte, asociaciones entre tipo de aprendizaje y de inteligencia mostraron que no existe relación entre aprendizaje teórico, aprendizaje pragmático y tipo de inteligencia verbal, donde esta última variable solo explicó en 0,4 el

Tabla 1. Porcentaje de estudiantes por cada tipo de aprendizaje, nivel y rendimiento académico.

\begin{tabular}{|c|c|c|c|c|c|c|c|}
\hline \multirow[b]{2}{*}{ Tipo de inteligencia } & \multicolumn{3}{|c|}{ Nivel de aprendizaje } & \multicolumn{3}{|c|}{ Rendimiento académico } & \multirow{2}{*}{$\begin{array}{l}\% \text { total } \\
\text { por tipo }\end{array}$} \\
\hline & Bajo & Medio & Alto & 2.00 & 3.00 & 4.00 & \\
\hline Inteligencia verbal & $60 \%$ & $16 \%$ & ${ }^{24}$ & $\begin{array}{l}16,7 \\
\%\end{array}$ & $\begin{array}{l}66,7 \\
\%\end{array}$ & $\begin{array}{l}16,6 \\
\%\end{array}$ & 52,2 \\
\hline $\begin{array}{c}\text { Inteligencia } \\
\text { lógico-matemática }\end{array}$ & $33 \%$ & $12 \%$ & $\begin{array}{l}55 \\
\%\end{array}$ & $0,0 \%$ & $\begin{array}{l}33,3 \\
\%\end{array}$ & $\begin{array}{l}66,7 \\
\%\end{array}$ & 26,1 \\
\hline Visual/Espacial & $52 \%$ & $20 \%$ & $\begin{array}{l}28 \\
\%\end{array}$ & $45 \%$ & $\begin{array}{l}35,0 \\
\%\end{array}$ & $\begin{array}{l}20,0 \\
\%\end{array}$ & 24,7 \\
\hline Kinestésico/Corporal & $\begin{array}{l}39,5 \\
\%\end{array}$ & $\begin{array}{l}25,5 \\
\%\end{array}$ & $\begin{array}{l}35 \\
\%\end{array}$ & $0,0 \%$ & $\begin{array}{l}75,0 \\
\%\end{array}$ & $\begin{array}{l}25,0 \\
\%\end{array}$ & 17,4 \\
\hline Musical/Rítmica & $\begin{array}{l}47,5 \\
\%\end{array}$ & $25 \%$ & $\begin{array}{l}23 \\
\%\end{array}$ & $\begin{array}{l}50,0 \\
\%\end{array}$ & $\begin{array}{l}37,5 \\
\%\end{array}$ & $\begin{array}{l}12,5 \\
\%\end{array}$ & 34,8 \\
\hline Intrapersonal & $5 \%$ & $65 \%$ & $\begin{array}{l}30 \\
\%\end{array}$ & $\begin{array}{l}22,2 \\
\%\end{array}$ & $\begin{array}{l}50,0 \\
\%\end{array}$ & $\begin{array}{l}27,8 \\
\%\end{array}$ & 78,3 \\
\hline Interpersonal & $27 \%$ & $53 \%$ & ${ }^{20}$ & $\begin{array}{l}12,5 \\
\%\end{array}$ & $\begin{array}{l}56,3 \\
\%\end{array}$ & $\begin{array}{l}31,2 \\
\%\end{array}$ & 69,6 \\
\hline
\end{tabular}

Fuente: elaboración propia. 
aprendizaje teórico $(r=0,006324555)$, en $2 \%$ el aprendizaje reflexivo $(r=0,014)$.

La inteligencia lógico-matemática explicó el aprendizaje teórico en 23,8 \%; el aprendizaje reflexivo, en $5 \%$; el aprendizaje activo, en $2 \%$. La inteligencia visual espacial explicó en $40 \%$ el aprendizaje teórico; aprendizaje pragmático, en $20 \%$; aprendizaje activo, en $13 \%$, aprendizaje reflexivo, en $12 \%$. Por su parte, la inteligencia kinestésico-corporal explicó en 50 \% el aprendizaje teórico; aprendizaje pragmático, en $11 \%$, con una relación negativa, y el aprendizaje activo lo explicó en $16 \%$.

Inteligencia musical explicó el aprendizaje pragmático en $26 \%$; aprendizaje reflexivo, 0,06 $\%$; aprendizaje activo, en $46 \%$; aprendizaje teórico, en $41 \%$. La inteligencia intrapersonal explicó en $16 \%$ (relación negativa) al aprendizaje pragmático; 35,2 \% el aprendizaje reflexivo; $40 \%$ al aprendizaje activo; 45,4\% aprendizaje teórico. La inteligencia interpersonal explicó en 0,2 \% el aprendizaje pragmático; $55 \%$, al aprendizaje reflexivo, y no existió relación alguna con el aprendizaje activo. En general, no se encontró relación significativa entre el tipo de inteligencia y el tipo de aprendizaje de los estudiantes.

Los resultados de la verificación de supuestos mostraron un valor del determinante de 0,0000136 , el KMO alcanzó el valor de 0,67 y la prueba de esfericidad de Barlett arrojó $\chi 2=632,72$ y $p=0,00$, niveles de pruebas que garantizaron la adecuación de datos para realizar del análisis factorial.

Se obtuvo una configuración tetrafactorial, en la cual, los pesos factoriales oscilaron entre $0,58 \mathrm{y}$ 0,9. El primer factor, estilo de aprendizaje, explicó un $41,2 \%$ de la varianza, compuesto por variables activo (carga $=0,67)$, reflexivo (carga $=0,8)$, teórico (carga $=0,6)$, pragmático $($ carga $=0,86)$. El segundo factor, tipo de inteligencia, explica aproximadamente el 38,8 \% de la varianza, compuesto por inteligencia verbal (carga $=0,81$ ), inteligencia lógico-matemática (carga $=0,72$ ), visual-espacial $($ carga $=0,83)$, kinestésico-corporal $($ carga $=79)$, musical rítmica (carga $=0,8$ ), intrapersonal (carga
$=0,81$ ), interpersonal (carga $=0,79$ ). La didáctica, como tercer factor, explicó aproximadamente el $10 \%$ de la varianza, asociado a recursos en el aula $(0,73)$, profesor $(0,76)$, motivación $(0,81)$, un cuarto factor, puntaje, explicó un 7,8 \% de la varianza, con variables rendimiento (carga $=0,89$ ), nivel de aprendizaje $(0,92)$, y factor conciencia de déficit con variable razonamiento abstracto $(0,9)$, sensibilidad $(0,71)$.

Una vez realizado el análisis factorial confirmatorio (figura 3), se observan cinco variables exógenas o factores: tipo de aprendizaje, estilo de aprendizaje, motivación, tipo de inteligencia y conciencia de déficit, además de quince variables endógenas asociadas al rendimiento académico del estudiante adulto.

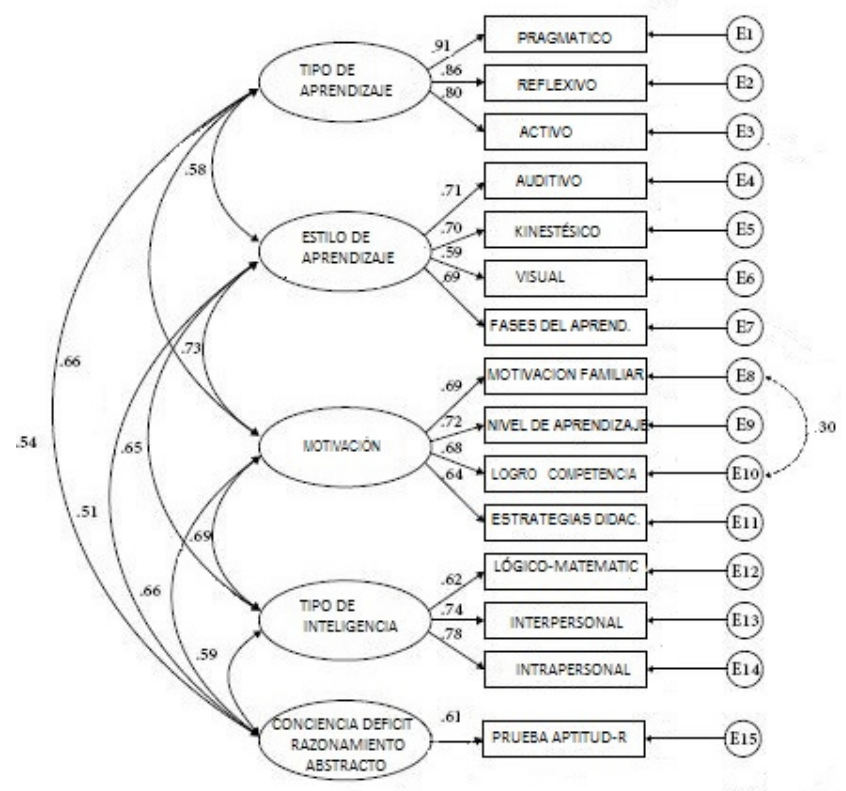

Figura 3. Modelo estructural confirmatorio factores asociados al rendimiento académico en adultos

Fuente: elaboración propia.

Se obtiene para el modelo valores satisfactorios $(>0,9)$ (Bentler, 1992) del non-normed fit index, $\mathrm{NNFI}=0,996 ; \mathrm{CFI}=0,998$ (comparative fit index); IFI = MFI =0,998; GFI (goodnes of fit index) igual a 0,981 e índice ajustado AGFI =0,971. De otra parte, índice de criterio, $\mathrm{AIC}=362,81$; índice de 
residuos, $R M R=0,027$, e índices de ajuste muestran que el modelo tomado obtuvo un buen ajuste a los datos.

\section{Discusión}

Determinar factores asociados al rendimiento académico en adultos permitió observar que el tipo de inteligencia, tipo y estilo de aprendizaje son factores también presentes en el modelo; sin embargo, nuevos factores se asocian a este proceso, como la motivación influida por la familia al estudiante adulto o la conciencia de déficit del adulto.

Estudiantes con edad mayor a 30 años, tuvieron dificultades con el aprendizaje visual, es decir, aprender por observación no daría buenos resultados, lo que dificultaría el aprendizaje de la estadística y la matemática, dado que, estas disciplinas manejan un alto lenguaje visual a través de gráficas y tablas. Preocupante el caso de quienes, según el test de aprendizaje visual, no evidencian este tipo de aprendizaje, lo cual supone una relación entre este factor y el rendimiento académico, y la búsqueda de otra estrategia didácti$\mathrm{ca}$, dado que los docentes enfatizan en aspectos gráficos.

Contrario a lo manifiesto por Laguzzi et al. (2013), los estudiantes adultos no prefieren el aprendizaje visual, activo y estilos secuenciales. Es recomendable entonces, realizar ejercicios para mejorar el aprendizaje visual ya que esta debilidad no favorece el aprendizaje de asignaturas como matemáticas, estadística, macro y microeconomía, importantes para la formación del administrador público.

Tal como lo plantea Arcavi (2003) al combinar las definiciones dadas por Serna (2015) y Thagard, P. (2012), desde una perspectiva de la multidimensionalidad y la complejidad, la visualización debe incorporarse en la metodologías de syllabus o planes de cursos de niños y jóvenes para potenciarles esta habilidad, de manera que, en edad adulta se constituya en una capacidad, potenciada como proceso y producto de creación, interpretación, uso y reflexión sobre imágenes, diagramas, en la mente, sobre papel o con herramientas tecnológicas, con el propósito de representar y comunicar información sobre el pensamiento y desarrollo de ideas previamente desconocidas y avanzar en la comprensión matemática y de análisis.

Así mismo, la inteligencia interpersonal estuvo presente en un alto porcentaje de estudiantes $(69,6 \%)$, explicada por el rol que desempeñan como trabajadores en el sector público o privado, o en sus propias microempresas, y que según la teoría de Gardner es propia de este tipo de personas (Roman y Murillo, 2011). De hecho, tal situación favorece el trabajo en equipo para mejorar el aprendizaje, porque son personas que se relacionan muy bien.

Similar a Krusche (2006) y Sabogal (2013), se observó que los canales sensoriales del adulto revisten importancia tanto en la percepción externa como en la interna, dado que, un objeto se puede observar y se puede imaginar. Este estilo depende de tres parámetros, cómo el individuo selecciona la información, cómo la organiza y cómo trabaja la información.

Se confirmó que considerar inteligencias, capacidades o fortalezas a las facultades desarrolladas en los estudiantes, resulta de suma utilidad para los profesores, ya que permite comprenderlos más y delinear actividades y estrategias metodológicas apropiadas para obtener los máximos resultados en el rendimiento académico. Además se encontró que la motivación que reciben en sus hogares incide de manera significativa con el rendimiento académico de los alumnos adultos, al igual que tener conciencia de déficit para fortalecer temáticas en las cuales presentaron dificultad.

Los resultados sugieren un nicho para el desarrollo de investigaciones en adultos y asociadas a factores psicosociales y de capacidad, u otras variables con potencial pragmático, para implementar estrategias o realizar intervenciones que lleven a la mejora de la calidad en la educación universitaria. 


\section{Conclusiones}

Factor motivación con variables endógenas asociadas a la familia y a las estrategias didácticas utilizadas por el profesor; así mismo, conciencia de déficit relacionado al razonamiento; y, estilos de aprendizaje, tipo de aprendizaje y tipo de inteligencia, los cuales explican el rendimiento académico y el logro de competencias en los estudiantes.

El rendimiento académico en estudiantes con edad mayor a 30 años, se relaciona con tener un tipo de aprendizaje auditivo, kinestésico, con preferencia al estilo de aprendizaje pragmático y reflexivo. Las inteligencias intrapersonal e interpersonal son los tipos de inteligencia más significativas en adultos, con un nivel de aprendizaje medio.

Estudiantes con inteligencia lógico-matemática lograron un alto nivel de aprendizaje y alto rendimiento académico en las áreas de matemática y estadística.

\section{Referencias Bibliográficas}

Alegret, M.; Espinosa, A.; Valero, S.; Vinyes-Junqué, G.; Ruiz, A.; Hernández, I.; et al. (2013). Cut-off scores of a brief neuropsychological battery (NBACE) for Spanish individual adults older than 44 years old. PLoS One, 8(2), 1-8

Alegret, M.; Espinosa, A.; Vinyes-Junqué, G.; Valero, S.; Hernández, I.; Tárraga, L.; et al. (2012). Normative data of a brief neuropsychological battery for Spanish individuals older than 49 . Journal Clinic Exp Neuropsychol, 34, 209-219.

Arcavi, A. (2003). The role of visual representations in the learning of mathematics. Educational Studies in Mathematics, 52(3), 215-241.

Bartual, T. y Poblet, M. (2009). Determinantes del rendimiento académico en estudiantes universitarios de primer año de Economía. Revista de Formación e Innovación Educativa Universitaria, 2(3), 172-181. Recuperado de: http:// refiedu.webs.uvigo.es/Refiedu/Vol2_3/ REFIEDU_2_3_6.pdf
Bentler, P. (1992). On the fit of models to covariances and methodology to the Bulletin. Psychological Bulletin, 112, 400-404.

Erazo, O. (2013). Caracterización psicológica del estudiante y su rendimiento académico. Revista Colombiana de Ciencias Sociales, 4(1), 23-41. Recuperado de: http:// www.funlam.edu.co/revistas/index.php/RCCS/article/ view/948/870

Fernández, N. (2012). Retención y persistencia estudiantil en instituciones de educación superior: una aproximación interdisciplinaria al concepto. Paradigma, 33(2), 63-88. Recuperado de: http://www.scielo.org.ve/scielo. php?pid=S1011-22512012000200004\&scrip$\mathrm{t}=$ sci_arttext

Garbanzo, G (2007). Factores asociados al rendimiento académico en estudiantes universitarios, una reflexión desde la calidad de la educación superior pública. Revista Educación, 31(1), 44-57.

Garbanzo, G. (2013). Factores asociados al rendimiento académico en estudiantes universitarios desde el nivel socioeconómico: un estudio en la Universidad de Costa Rica. Revista Electrónica Educare, 17(3), 57-87. Recuperado de: http://www.revistas. una.ac.cr/index.php/ EDUCARE/article/view/5258/5069

Gardner, H. (1983). Estructuras de la mente. México: Fondo de Cultura Económica.

Horton, A, y Hartlage, L. (2010). The Handbook of Forensic Neuropsychology. Nueva York: Springer Publishing Company.

Krusche, H. (2006). La rana sobre la mantequilla: fundamentos de la programación neurolingüística. Málaga, España: Sirio.

Laguzzi, J.; Bernardi, S.; Araujo, A.; Ventura, A. y Vigliano, F. (2013). Result 20. Learning-styles of students of veterinary medicine in the National University of Rosario, Argentina. Informe técnico. Santiago de Chile: Unesco.

Martin, A.; Wilson, R.; Arief, G. y Ginns, P. (2013). Academic Momentum at University/College: Exploring the Roles of Prior Learning, Life 
Experience, and Ongoing Performance in Academic Achievement Across Time. The Journal of Higher Education, 84(5), 640-674. Doi: http://dx.doi.org/10.1353/jhe.2013.0029

Martínez, J.J.; Vergel, M. y Zafra, S.L. (2016). Comportamiento juvenil y desarrollo de competencias prosociales. Bogotá: Ibáñez.

Mayora, C. y Fernández, N. (2015). Locus de control y rendimiento académico en educación universitaria: una revisión bibliográfica. Revista Electrónica Educare Electrónica, 19(3), 1-23. Doi: http://dx.doi.org/10.15359/ree.19-3.16

Muldoon, R. y Weisberg, M. (2011). Robustness and idealization in models of cognitive labor. Synthese, 183, 161-174.

Ortega, G.; Alegret, M.; Espinosa, A.; Ibarria, M.; Cañabate, P. y Boada, M. (2014). Valoración de las funciones visuoperceptivas y visuoespaciales en la práctica forense. Revista Española de Medicina Legal, 40, 83-95.
Román, M. y Murillo, F. (2011). América Latina: violencia entre estudiantes y desempeño escolar. Revista CEPAL, 104, 37-54.

Serna, E. (2015). Construcción disciplinar del conocimiento científico. Revista Científica, 22, 111-128. Doi: 10.14483/udistrital.jour. RC.2015.22.a9

Sabogal, A. (2013). Learning styles in the instructional design of a virtual course in indigenous language. Presentado en la XII Conferencia Iberoamericana en Sistemas, Cibernética e Informática, Decimo Simposio Iberoamericano en Educación, Cibernética e Informática, SIECI 2013. Recuperado de: www.scopus.com Thagard, P. (2012). The cognitive science of science: Explanation, discovery, and conceptual change. Cambridge: MIT Press.

Wechsler, D. (1999). Escala de Inteligencia de Wechsler para adultos-III. Manual de Aplicación y corrección. Madrid: TEA Ediciones. 